\title{
Aplicación de técnicas estadísticas en investigaciones académicas con enfoque zootécnico
}

\section{Application of statistical techniques in academic research with zootechnical approach}

\author{
Liz Mariela Centurión-Insaurralde ${ }^{1}$ (D) y Oscar Roberto Martínez-López ${ }^{2 *}$ (iD \\ ${ }^{1}$ Facultad de Ciencias Exactas y Naturales, Universidad Nacional de Asunción. San Lorenzo. Paraguay. \\ ${ }^{2}$ Centro Multidisciplinario de Investigaciones Tecnológicas, Universidad Nacional de Asunción. San Lorenzo. Paraguay. \\ Correo electrónico: robertomartinezlo@vet.una.py
}

\section{RESUMEN}

El empleo de las herramientas estadísticas en investigaciones científicas y académicas es fundamental, porque sustenta las conclusiones con base en los resultados obtenidos. Con el objetivo de determinar la aplicación de técnicas estadísticas en investigaciones según la especie zootécnica estudiada, se efectuó un análisis exhaustivo considerando 845 tesis de grado con orientación en producción animal, en el periodo comprendido entre el 2010 y 2016 en la mayor universidad pública del Paraguay. En la mayoría de los trabajos (95,86 \%), se observó el empleo de al menos una herramienta estadística para el análisis de los datos. La especie zootécnica mayormente estudiada lo constituyeron los bovinos $(61,18 \%)$. En lo concerniente al tipo de técnica estadística empleada, para las especies de aves, bovinos, conejos, ovinos, porcinos y multiespecies, destacó el análisis de varianza, para las demás se acentuó la estadística descriptiva.

Palabras clave: Técnicas estadísticas; zootecnia; especie zootécnica; tesis de grado

\begin{abstract}
The use of statistical tools in scientific and academic research is fundamental, because it supports the conclusions based on the results obtained. In order to determine the application of statistical techniques in research according to the zootechnical species studied, an exhaustive analysis was carried out considering 845 Grade Thesis with orientation in animal production in a period between 2010 and 2016 in the largest public university in Paraguay. It was observed the use of at least one statistical tool for the analysis of data in most of the works $(95.86 \%)$. The most studied zootechnical species were bovines $(61.18 \%)$, regarding the type of statistical technique used, for bird, bovine, rabbit, ovine, porcine and other species, the variance analysis stood out for the others the descriptive statistics was accentuated.
\end{abstract}

Key words: Statistical techniques; zootechnics; zootechnical species; grade thesis 


\section{INTRODUCCIÓN}

En Paraguay, el área agropecuaria constituye un sector fundamental en la economía, donde gran parte de la misma se apoya en la producción pecuaria, específicamente en la bovina (Bos taurus y Bos indicus). Sin embargo, la producción avícola (Gallus domesticus) y la porcina (Sus scrofa domesticus) han experimentado un aumento, asimismo, la cría de cabras (Capra aegagrus hircus), ovejas (Ovis orientalis aries) y conejos (Oryctolagus cuniculus) [8].

En estudios sobre procesos zootécnicos o producción animal (PA), es fundamental conocer si las situaciones acontecidas son debidas al azar o si corresponden a hechos causales, en consecuencia se requiere el empleo de herramientas estadísticas que permitan obtener esa discriminación, a efectos de una comprensión cabal sobre la naturaleza del fenómeno estudiado. Las investigaciones científicas en el área de la zootecnia son transcendentales para el desarrollo de planes y/o estrategias referente a diversos aspectos que hacen a la misma, como las condiciones sanitarias, la genética y la nutrición animal. En ese contexto, las instituciones académicas, y más concretamente, las universidades poseen una responsabilidad primordial con la sociedad, debido a que una de sus funciones es la producción del conocimiento a través de la investigación científica.

En una universidad pública del Paraguay, en el área de la PA, las investigaciones de grado o tesis son un requerimiento para la obtención de un grado académico. Independientemente de esa finalidad, una tesis consiste en la realización de una investigación para la consolidación del conocimiento sobre el método científico, en ese sentido, implica el desarrollo de una investigación con un sustento teórico, con métodos y procedimientos válidos, para lo cual es insoslayable la aplicación de técnicas estadisticas. La participación de estudiantes en investigaciones de esta índole podría constituirse en un acercamiento primario y único al desarrollo de una investigación con rigor científico.

Estos trabajos de investigación de grado, básica o aplicada, con orientación en PA, no se considera como un eje indispensable el conocimiento sobre la naturaleza de las distintas técnicas estadisticas y su efecto-respuesta en los resultados, lo cual podria conducir a diferentes tipos de sesgos. En esa linea de análisis, Echavarría y col. [2], en una investigación sobre los métodos estadísticos usados en los trabajos de grado y tesis realizados en una Facultad de Ciencias Agropecuarias, encontraron algunas falencias como la inclusión de resultados estadísticos que no contribuían en absoluto al cumplimiento de los objetivos, tales como la omisión de resultados relacionados intrínsecamente con los objetivos, entre otros. Según los autores, las fallas encontradas son producto de una exigua claridad de la función que tienen las herramientas estadísticas para la consecución de los objetivos de una investigación.

En la presente investigación, se abordó de manera metódica y sistemática, la identificación y la clasificación de las herramientas estadísticas utilizadas en diferentes especies zootécnicas en trabajos de investigaciones académicas con orientación en PA, en una universidad pública del Paraguay.

\section{MATERIALES Y MÉTODOS}

La investigación se desarrolló en Paraguay, en la mayor universidad pública del país, específicamente en dos unidades académicas localizadas en el Departamento Central, de la ciudad de San Lorenzo. El levantamiento de los datos se efectuó durante los años 2017 y 2018, analizando un total de 845 tesis con orientación en PA en un período comprendido entre el 2010 y el 2016, respectivamente.

En cada una de las tesis se observó la especie zootécnica abordada, considerándose la siguiente clasificación: acuicultura y pesca, caprino, ovino, conejo, ave, porcino, equino (Equus caballus) y multiespecie. Es preciso aclarar que, la categoría acuicultura y pesca, no es una especie zootécnica y fue considerada bajo esta denominación a efectos de agrupar e incluir a todos los organismos vivos acuáticos, que fueron objetos de estudio y análisis en las tesis de grado (TdG). La variable: empleo de herramientas estadísticas fue categorizada en dos niveles (si y no). El número de herramientas estadísticas fue organizado en tres categorías (una, dos y tres herramientas). Finalmente, el tipo de herramienta empleada, agrupado en ocho estratos: estadística descriptiva (ED), T-Student para muestras independientes ( $\mathrm{TI})$, T-Student para muestras apareadas (TA), Ji-cuadrado (JI), análisis de regresión (AR), análisis de varianza (AV), coeficiente de correlación de Pearson (CP) y otras (OT). Los datos fueron analizados mediante el software estadístico R Project for Statistical Computing [13], aplicando estadística descriptiva y la técnica multivariante, análisis cluster o de conglomerados, utilizada para la clasificación automática de los datos.

\section{RESULTADOS Y DISCUSIÓN}

En la TABLA I se presenta la distribución de las frecuencias absolutas y porcentuales de las TdG según especies zootécnicas. La especie dominante correspondió a bovinos, aglomerando el $61 \%$ del total de tesis analizadas. Estos resultados evidencian un posible interés por parte de los estudiantes en realizar investigaciones en donde la especie objeto de estudio sean bovinos. Además, se podría suponer que constituye un indicador de prioridad el desarrollo de actividades de investigación que involucre a este grupo de especies, en las unidades académicas consideradas. Este resultado coincide parcialmente con lo evidenciado por San Martín y Pacheco [16], analizando tesis en el área de veterinaria. Los mismos constataron que además de los bovinos, la especie canina (Canis familiaris) también presentó una alta frecuencia de estudio. En este sentido, es menester mencionar que las tesis analizadas conciernen al área de PA, si se hubiesen considerado trabajos del área de medicina veterinaria probablemente los resultados serían distintos.

El hecho de que los bovinos fueron mayormente estudiados en las investigaciones académicas, podría deberse entre otros factores, a los económicos, esto es debido a que gran parte de la economía paraguaya se apoya en la producción bovina. Al respecto, Ortega y García [11] manifiestan que, los bovinos constituyen una especie zootécnica que ostentan una variabilidad genética importante y que a través de investigaciones pueden ser estudiadas para obtener un mejoramiento en el animal y, maximizar el rendimiento de los parámetros productivos de los mismos.

En la FIG. 1 se presenta el agrupamiento de las TdG según especie zootécnica. Como se puede apreciar, el resultado generado mediante el análisis cluster conduce a afirmaciones equivalentes en observancia a lo obtenido en la TABLA I. Se visualiza que los equinos, caprinos, acuicultura y pesca, conejos, y multiespecies conforman un solo agrupamiento, las cuales conciernen a las especies menormente consideradas en las investigaciones 
TABLA I

Frecuencias absolutas y porcentuales de las tesis según especie zootécnica

\begin{tabular}{ccc}
\hline Especie zootécnica & Número de Tesis & Frecuencia (\%) \\
\hline Acuicultura y pesca & 29 & 3,43 \\
Ave & 87 & 10,30 \\
Bovino & 517 & 61,18 \\
Caprino & 19 & 2,25 \\
Conejo & 30 & 3,55 \\
Equino & 24 & 2,84 \\
Ovino & 72 & 8,52 \\
Porcino & 44 & 5,21 \\
Multiespecie & 23 & 2,72 \\
\hline Total & 845 & 100 \\
\hline
\end{tabular}

Especies Zootécnicas

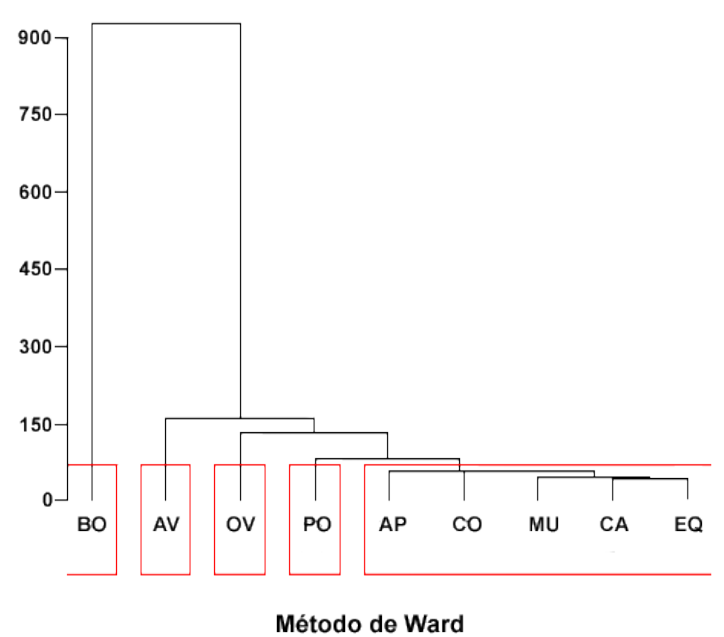

FIGURA 1. Agrupamiento de las tesis de grado por especies zootécnicas utilizando el Método de Ward. BO: bovinos; AV: aves; OV: ovinos; PO: porcinos; AP: acuicultura y pesca; CO: conejos; MU: Multiespecies; CA: caprinos; EQ: equinos

académicas analizadas (TABLA I). El dendrograma sugiere la conformación de cinco (5) grupos, en donde tanto los bovinos, como las aves, los ovinos y los porcinos forman grupos de manera independiente.

En la TABLA II, se observa la distribución del número de tesis y los valores porcentuales de la variable empleo de herramientas estadísticas en las TdG. A partir de la misma, se constató que en aproximadamente el $96 \%$ del total fueron empleadas al menos una herramienta estadística para el análisis de los datos. En este sentido, González de Dios y col. [4], mencionan la importancia del empleo de la herramienta estadística en una investigación, afirmando que constituye un aspecto esencial para el sustento del rigor científico, permitiendo de esta forma la generación de conclusiones válidas.

TABLA II

Frecuencias absolutas y porcentuales del empleo de las herramientas estadísticas en las tesis de grado

\begin{tabular}{ccc}
\hline $\begin{array}{c}\text { Empleo de herramientas } \\
\text { estadísticas }\end{array}$ & $\begin{array}{c}\text { Número } \\
\text { de Tesis }\end{array}$ & Frecuencia (\%) \\
\hline Si & 810 & 95,86 \\
No & 35 & 4,14 \\
\hline Total & 845 & 100 \\
\hline
\end{tabular}

También se destaca que del total de estudios en 35 de los trabajos, no se utilizó ninguna técnica de análisis estadístico representando el $4,14 \%$ del total (TABLA II). Al comparar estos resultados con los obtenidos en un estudio previo, se observa que el porcentaje de trabajos que no emplearon técnicas estadísticas es cinco veces mayor que lo evidenciado en la presente investigación [9]. Cabe señalar que, de estas 35 tesis, tres de ellas pertenecieron a análisis experimentales y 32 , es decir, la mayoría, fueron evaluaciones y/o análisis económicos, entre las mismas, los temas abordados consistieron por ejemplo en: el porcentaje de rentabilidad que se puede alcanzar en una inversión en un periodo de tiempo, evaluaciones de sistemas de producción, de suplementación, entre otros. Si bien, fueron empleadas técnicas de análisis para la generación de conclusiones, no se aplicaron técnicas estadísticas que fundamenten a estas. Al respecto, Vásquez-Bedoya y col. [17] destacan algunas técnicas económicas y estadísticas que pueden utilizarse en investigaciones de tipo económico, como por ejemplo los Modelos Deterministas y Estocásticos, las Funciones Polinomiales, las Diferencias de Primer Orden, entre otros.

El procedimiento estadístico contribuye a discriminar si las rentabilidades obtenidas a partir de un sistema productivo, corresponden a hechos casuales o causales. Su empleo es esencial debido a que apoyan las evaluaciones económicas realizadas, que de manera aislada podrían conducir a una toma de decisiones no tan acertada. Vale decir que, en un proceso biológico, en este caso en el área de la PA interesa saber si el fenómeno estudiado es de carácter aleatorio o si tiene un sustento característico, particular o frecuente de ese hecho.

Las investigaciones en el ámbito de la zootecnia, desde un enfoque económico son importantes para evaluar la rentabilidad de los sistemas de producción. Sin embargo, debe existir una sinergia entre los procesos biológicos, los económicos y los matemáticos para explicar la dinámica de este tipo de prácticas zootécnicas [7].

Las TdG se constituyen en investigaciones académicas y se caracterizan por el desarrollo de un análisis crítico, metódico y lógico, a partir de la identificación de un problema inscrito en un área específico [12]. Se puede decir que éstas implican el sustento de lo que se investiga, se analiza y se concluye sobre un pilar denominado rigor científico. Con base en la literatura 
consultada se pudo visualizar que, en una universidad extrajera homóloga a la universidad pública considerada en el estudio, la Universidad de la República Oriental del Uruguay, en una carrera muy afín a la considerada en este trabajo, se da la opción al estudiante para seleccionar la modalidad de la TdG a realizar, estas modalidades consisten en: ensayo experimental, estudio de caso y revisión monográfica, en donde cada una de las mismas tienen características propias y enfoques distintos en cuanto al desarrollo del trabajo de investigación.

Atendiendo lo expresado, estas modalidades permitirían al estudiante orientar su trabajo de tesis sin que se genere confusión en él, en cuanto a la realización de un trabajo de investigación con rigor científico, teniendo en cuenta que desde el inicio ya tendrá conocimiento de que su trabajo carecerá o no, de rigor científico, dependiendo de la modalidad seleccionada.

Siguiendo en la misma línea, se podría indicar que, si un estudiante desea realizar una investigación enfocada al área económico, podría optar por la modalidad estudio de caso. En este punto, es importante destacar que no existe una distinción de modalidades en los trabajos de investigación evaluados, éstos se encuentran bajo una misma denominación: TdG, lo cual implica una metodología de investigación rigurosa, la cual lleva implícito la utilización de herramientas estadísticas.

En la TABLA III se visualiza que, en la mayoría de los trabajos se empleó un solo método estadístico para el análisis de los datos, representando aproximadamente el $83 \%$ del total. Sin embargo, en un $15,68 \%$ fueron aplicadas dos herramientas y en tan solo $1 \%$, tres. Es oportuno indicar que, existen varios métodos estadísticos para el análisis de un banco de datos, la elección de uno u otro dependerá del objetivo propuesto, de las hipótesis establecidas y de las características de las variables, según Siegel y Castellan [15] estas consideraciones permiten elegir la técnica más óptima.

En un estudio previo desarrollado por Massip-Nicot y col. [6] discriminaron en una escala del 1 al 6 , el: número de procedimientos estadísticos aplicados en los artículos originales publicados en una revista científica, los resultados concluyeron que en aproximadamente el $53 \%$ fueron empleadas solamente una técnica estadística, en $22 \%$ dos herramientas y en $14 \%$, tres; resultados que coinciden con lo obtenido por Romani y col. [14] en otra investigación similar. A diferencia de lo evidenciado en este trabajo, hubo una mayor cantidad herramientas empleadas en los artículos de investigación analizados por estos autores.

TABLA III

Frecuencias absolutas y porcentuales del número de herramientas estadísticas empleadas en las tesis de grado

\begin{tabular}{ccc}
$\begin{array}{c}\text { Herramientas } \\
\text { estadísticas }\end{array}$ & Número de Tesis & Frecuencia \% \\
\hline Una herramienta & 675 & 83,33 \\
Dos herramientas & 127 & 15,68 \\
Tres herramientas & 8 & 0,99 \\
\hline Total & 810 & 100 \\
\hline
\end{tabular}

El empleo de distintos procedimientos estadísticos en un mismo conjunto de datos, siguiendo las atenciones expresadas por Siegel y Castellan [15], proporcionarían alternativas que permitan una visualización más completa del comportamiento del proceso biológico, posibilitando una interpretación objetiva de los resultados generados desde diferentes aristas. Es importante recalcar que, debe existir una simbiosis entre la estadística y los procesos zootécnicos para la formulación de conclusiones válidas. Al respecto, Montes de Oca y col. [9] señalan que, en los trabajos de investigación en el ámbito de la PA, los procedimientos estadísticos deben estar anidados, tanto en la metodología de investigación propiamente como en el diseño experimental. Asimismo, Barreto-Villanueva [1] menciona que en los distintos campos del conocimiento, la estadística no puede verse separada de la investigación científica, es más, manifiesta que en algunos ámbitos es imprescindible el proceder estadístico.

En la TABLA IV se observan las frecuencias absolutas de las distintas herramientas estadísticas empleadas en los trabajos de investigación según especies zootécnicas. Las categorías de la variable están dispuestas en columnas, presentando un total de ocho clasificaciones. Con base a lo expuesto se puede decir que, para las especies equinos y, acuicultura y pesca, la estadística descriptiva fue la técnica de mayor empleo en comparación con las demás. Sin embargo, también se destacó la utilización de esta herramienta estadística en las tesis donde constituyeron otras especies las unidades de observación.

Por otro lado, para las demás especies consideradas, claramente resaltó el empleo del AV presentando mayores frecuencias de utilización. En ese sentido, en trabajos donde fueron estudiados bovinos, se constató una mayor heterogeneidad de técnicas de análisis. Sin embargo, como se mencionó anteriormente, el AV fue superior en comparación con las demás. Otras técnicas acentuadas pero, con frecuencias menores y decrecientes correspondieron al análisis de regresión, al Ji-cuadrado y al T-Student para muestras independientes.

Al respecto Martínez-López y col. [5], manifiestan la importancia del empleo de las herramientas estadísticas en investigaciones en el ámbito de la PA. En una investigación desarrollada por los mismos, valoraron la utilización de la correlación para identificar relaciones entre variables morfométricas en especies zootécnicas, específicamente en bovinos, considerando al grupo genético, Pampa Chaqueño. Igualmente, Galindo-Gil y col. [3] destacan la utilización de correlaciones estadísticas para evaluar relaciones entre características morfológicas y ecológicas en especies de lagartijas (Sceloporus aeneus, Sceloporus bicanthalis, Sceloporus clarkii, Sceloporus formosus, Sceloporus gadoviae, Sceloporus grammicus, Sceloporus horridus, Sceloporus jalapae, Sceloporus jarrovi, Sceloporus magister, Sceloporus megalepidurus, Sceloporus mucronatus, Sceloporus nelsoni, Sceloporus occidentalis, Sceloporus poinsetti, Sceloporus spinosus, Sceloporus torquatus, Sceloporus undulatus, Sceloporus utiformis y Sceloporus variabilis); los autores mencionan que investigaciones de esta índole son importantes para obtener respuestas adaptativas, debido a que estas especies pueden ser encontradas en diversos hábitats.

Los resultados encontrados en este trabajo, coinciden en cierta medida con lo obtenido por Montes de Oca y col. [9], los mismos aducen que los procedimientos estadísticos predominantes en los artículos analizados en el área de PA concernieron a técnicas elementales, clasificando en esta categoría a la estadística 
TABLA IV

Frecuencias absolutas de las herramientas estadísticas empleadas en tesis de grado según especie zootécnica

\begin{tabular}{ccccccccc}
\hline \multirow{2}{*}{$\begin{array}{c}\text { Especie } \\
\text { zootécnica }\end{array}$} & ED & TI & TA & JI & AR & AV & CP & OT \\
\hline Acuicultura y pesca & 13 & 5 & 0 & 2 & 0 & 5 & 0 & 0 \\
Ave & 13 & 15 & 1 & 0 & 9 & 59 & 0 & 0 \\
Bovino & 86 & 59 & 11 & 63 & 77 & 277 & 15 & 8 \\
Caprino & 8 & 8 & 0 & 2 & 0 & 4 & 0 & 2 \\
Conejo & 1 & 8 & 0 & 4 & 0 & 17 & 1 & 0 \\
Equino & 21 & 1 & 0 & 2 & 0 & 0 & 0 & 0 \\
Multiespecie & 3 & 1 & 0 & 0 & 10 & 16 & 2 & 0 \\
Ovino & 14 & 13 & 4 & 14 & 5 & 25 & 2 & 2 \\
Porcino & 8 & 10 & 2 & 1 & 1 & 19 & 3 & 1 \\
\hline
\end{tabular}

ED: estadística descriptiva; TI: t-Student para muestras independientes; TA: t-Student para muestras apareadas; JI: Ji-cuadrado; AR: análisis de regresión; AV: análisis de varianza; CP: coeficiente de correlación de Pearson; OT: otras, análisis factorial de correspondencias, Kruskal-Wallis, Wilcoxon, prueba exacta de Fisher; coeficiente de correlación de Spearman, Mann Whitney, t-Student para una muestra, análisis de componentes principales

descriptiva, al AV, a la regresión lineal simple, al t-Student, a las pruebas no paramétricas, entre otros. Sin embargo, en un estudio desarrollado por Montilla-Peña [10], al analizar artículos publicados en la Revista Zootecnia Tropical del Instituto Nacional de Investigaciones Agrícolas, evidenció una mayor aplicación de métodos multivariados, lo cual no pudo visualizarse en este trabajo. Los métodos multivariados, no tuvieron mucha representatividad; entre los mismos se destacaron aplicaciones del análisis factorial de correspondencia y del análisis de componentes principales, agrupadas en la categoría OT (TABLA IV).

\section{CONCLUSIONES}

Las TdG de hecho, constituyen un elemento fundamental en la formación de profesionales del agro y evidencia la primera aproximación del técnico al método científico.

En la mayoría de las TdG se observó el empleo de al menos una herramienta estadística para el análisis de los datos, asimismo se constató un mayor número de trabajos que incluye a bovinos como unidades de estudio.

En cuanto al tipo de técnicas estadísticas empleadas, para las especies de aves, bovinos, conejos, ovinos, porcinos y multiespecies, la herramienta de mayor aplicación fue el AV, para las demás destacó el empleo de la estadística descriptiva.

El uso del análisis clúster resultó muy útil para la visualización y análisis de la información, al permitir una adecuada identificación de las especies zootécnicas sujetas a estudio en las TdG en el periodo evaluado.

\section{AGRADECIMIENTOS}

Los resultados de este trabajo derivan de una tesis de Maestría del Programa Paraguayo para el Desarrollo de la Ciencia y la Tecnología (PROCIENCIA), financiado por el Consejo Nacional de Ciencia y Tecnología (CONACYT) y la Facultad de Ciencias Exactas y Naturales de la Universidad Nacional de Asunción (FACEN-UNA).

\section{REFERENCIAS BIBLIOGRÁFICAS}

[1] BARRETO-VILLANUEVA, A. El progreso de la Estadística y su utilidad en la evaluación del desarrollo. Papeles de población. 18(73): 241-271. 2012.

[2] ECHAVARRÍA, H.; CORREA, G.; PATIÑO, J. F.; ACOSTA, J. J.; RUEDA, J. A. Evaluación de métodos estadísticos utilizados en trabajos de grado y tesis de los programas de la Facultad de Ciencias Agropecuarias, en un periodo de tres años. Rev. Fac. Nac. Agron. Medellín. 59(2): 3565-3580. 2006.

[3] GALINDO-GIL, S.; RODRÍGUEZ-ROMERO, F.; VELÁZQUEZRODRÍGUEZ, A. S.; MORENO-BARAJAS, R. Correlaciones Morfológicas entre la Forma de la Cabeza, Dieta y uso de Hábitat de Algunos Sceloporus de México: Un Análisis Cuantitativo. Int. J. Morphol. 33(1): 295-300. 2015.

[4] GONZÁLEZ-DE-DIOS, J.; GONZÁLEZ-MUÑOZ, M.; ALONSOARROYO, A.; ALEIXANDRE-BENAVENT, R. Comunicación científica $(\mathrm{XVI})$. Conocimientos básicos para leer (y escribir) un artículo científico (3): Material, métodos y Resultados. Acta Pediatr. Esp. 72(9): 203-208. 2014.

[5] MARTÍNEZ-LÓPEZ, O. R.; NÚÑEZ, L.; CASTRO, L.; RODRÍGUEZACOSTA, M. I.; ÁLVAREZ-MARTÍNEZ, R.; FLORENTÍN, A.; RAMÍREZ, L.; PEREIRA, W. Uso de correlación estadística para el estudio morfométrico de bovinos para carne: caso pampa chaqueño. Compend. Cien. Vet. 4(2): 26-32. 2014. 
[6] MASSIP-NICOT, J.; SOLER-CÁRDENAS, S.; TORRESVIDAL, R. M.. Uso de la estadística en la Revista Cubana de Higiene y Epidemiología, 1996-2009. Rev. Cub. Hig. Epidemiol. 49(2): 276-291. 2011.

[7] MESA-GRANDA, M.; BOTERO-AGUIRRE, M. La cachama blanca (Piaractus brachypomus), una especie potencial para el mejoramiento genetico. Rev. Colomb. de Cien. Pec. 20(1): 79-86. 2007.

[8] MINISTERIO DE AGRICULTURA Y GANADERÍA (MAG). 2004. Informe pais sobre la situación de los recursos zoogenéticos del Paraguay. Asunción, Paraguay. En linea: http://www.fao.org/3/a-bc250s.pdf. 10.03.2017.

[9] MONTES-DE-OCA, R.; UÑA-IZQUIERDO, F.; VIERA, G. G.; CARDOSO, G.P.; AVILÉS-MERENS, R. Aplicación de la estadística, el diseño experimental y software en artículos de la Revista de Producción Animal. Rev. Prod. Anim. Esp: 35-40. 2007.

[10] MONTILLA-PEÑA, L. J. Analysis of the scientific production of articles of Journal Tropical Zootecnia of the National Institute for Agricultural Research (2006-2013). 2016. Biblios. Perú. En linea: https://doi.org/gvf3. 01.12.2018.

[11] ORTEGA, J.; GARCÍA, L. El genoma bovino, métodos y resultados de su análisis. Rev. MVZ Córdoba. 16(1): 24102424. 2011.
[12] RAMIRO-HERNÁNDEZ., M.; CRUZ-ARANDA., E. La tesis en los cursos de especialización en Medicina. Rev. Med. Inst. Mex. Seguro Soc. 54: S228-S229. 2016.

[13] R CORE TEAM. R: A language and environment for statistical computing. 2018. R Foundation for Statistical Computing, Vienna, Austria. En Línea: https://www.R-project.org/. 01.11.2018.

[14] ROMANI, F.; MARQUEZ, J.; WONG, P. Uso de los métodos estadísticos en artículos originales de cinco revistas biomédicas peruanas. Periodo 2002-2009. Rev. Peruana Epidemiol. 14(2): 153-160. 2010.

[15] SIEGEL, S.; CASTELLAN, N. J. Medidas de Asociación no paramétricas. En: Estadística no paramétrica: aplicada a las ciencias de la conducta. 4a Ed. Trillas, México. 437 pp. 1995.

[16] SAN-MARTIN, F.; PACHECO, J. Análisis bibliométrico de las tesis de pregrado de la Facultad de Medicina Veterinaria de la Universidad Nacional Mayor de San Marcos en el periodo 2001-2006. RIVEP. 19(1): 82-92. 2008.

[17] VÁSQUEZ-BEDOYA, F. A.; RESTREPO-OCHOA, S. I.; LOPERA-SIERRA, J. F. Una revisión crítica de las técnicas de filtrado para la teoría de los ciclos económicos reales. Cuadernos de Economía. 29(53): 119-153. 2010. 